details of the characteristics of the many commercial grades of each type of fibre are included. It is perhaps inevitable that stress is laid on American research and practice, although reference is made to many British papers.

The book is extremely well written and presented; the illustrations are good, the printing very clear, and the binding and quality of paper are such as to make most English authors envious. All who are interested in textiles, whether they are research workers, manufacturers, salesmen, directors, teachers or students should find this volume particularly valuable, for it is probably the most important publication on textiles which has appeared in the last twenty years. The authors are to be congratulated on their achievement.

C. S. WHEWELL

\section{ELECTRONICS IN INDUSTRY}

Electronics for Industry

By W. I. Bendz, with the assistance of C. A. Scarlott. Pp. $x+501$. (New York: John Wiley and Sons, Inc.; London: Chapman and Hall, Ltd., 1947.) 5 dollars.

$\mathrm{T}$ HE purpose of this book is to introduce the engineer who has had no special training in electronics to the fundamental principles involved in them, and the means of their practical application. The latter include a very wide range: photo-electric devices, high-power rectifiers, high-frequency heating, and various types of control circuits. The diagrams are mostly basic circuits illustrating the methods used, and so are not confused by minor details, though some actual eircuits are given.

On the theoretical side, the book opens with a very clear introduction to as much of modern atomic physics as is necessary for the understanding of the action of practical electronic devices ; this is material on which the average engineer is often sadly deficient.

The characteristics of the various types of valves are then dealt with, particular attention being given to the form of discharge in gas-filled valves, and there are many illustrations of actual electrode systems. The nomenclature throughout is the American standard, which differs from that in use in Britain; but the meaning of each expression is fully defined. Attention is then given to rectifiers of all types, from the small power pack for ordinary valve circuits up to the high-power three-phase mercury vapour type, with constructional details of the latter. Considerable attention is given to various types of firing circuits; but distortion of wave form is only slightly dealt with. In the chapter on amplification the basic principles are clearly dealt with, the work being entirely confined to medium-frequency working, no reference being made to the special difficulties which arise with high-and ultra-high-frequency working. A chapter on oscillators follows, all the normal circuits including dynatron and beat-frequency circuits being given, as well as a short description of the piezo-electric crystal.

After a short chapter on modulated signals, the rest of the book is given up to the description (including circuit diagrams) of all types of commercial electronic applications, classified under various headings: and this part contains much information which, up to the present, has been difficult to get in collected form without reference to specialized papers.
Taken as a whole, the book should be of great value to the user of electronic devices; it is not intended for those who have to design them, though throughout there are many references to more detailed information, including a bibliography at the end of each chapter.

J. HOLIINGWORTH

\section{ATOMS FOR THE LAYMAN}

\section{The Atom and its Energy}

By Prof. E. N. da C. Andrade. Pp. xii $+196+12$ plates. (London: G. Bell and Sons, Ltd., 1947.) 10s. net.

\section{Meet the Atoms}

A Popular Guide to Modern Physics. By O. R. Frisch. Pp. xiv +226 . (London: Sigma Books, Ltd., 1947.) 12s. $6 d$. net.

$\mathrm{P}$

ROF. ANDRADE is an old hand at explaining the mysteries of atomic structure; his "Structure of the Atom" was the standard text in the days when electrons circulated on nicely calculable orbits around the nuclei that Nature provided for our study. The electrons have long since abandoned their tramway-like orbits and, like buses in a fog, have a mere probability of being somewhere near a given route; new kinds of nuclei can be manufactured at will in great variety; and we have learned how to use, or misuse, the immense energy of nuclear forces.

In "The Atom and its Energy", Prof. Andrade marshals the modern array of atomic facts and theories with his usual skill, illuminates them by ingenious analogies, and spices the dish with a literary flavour; though the reviewer's metaphors may be mixed, the author's are not, and they magnify the atoms to visible size with amazingly little distortion.

Prof. Frisch, in "Meet the Atoms", follows the more informal plan of taking the reader into the laboratory and explaining how experiments in nuclear physics are devised, performed and interpreted. The apparatus is there, real and life-sized, and there is no need to magnify it; but when an analogy can help in interpreting the observations, Prof. Frisch does not hesitate. For example, he compares the dual wave and particle aspects of light to the appearance of an animal that everyone's right eye sees as a dog and everyone's left eye sees as a cat; he remarks that in time we would get used to this and would cease to worry about which it 'really was'. Such analogies, crude though they may be, are in harmony with the atmosphere of a real laboratory, where the apparatus and the conversation are not always highly polished. Prof. Andrade's book belongs rather more to the study, and has both gained and lost by its slight detachment from laboratory details.

The cat-and-dog analogy does not apply to this pair of books; each author sees the atoms clearly with both eyes, and explains them to the reader in his own way. The books are not, of course, written for the expert, but they will hold the interest of physicists as well as of the less-specialized public for whom they are intended. They are books about atoms and not primarily about atomic energy; Prof. Frisch deliberately says very little about that subject, and Prof. Andrade is not at his best when dealing with its technical and scientific aspects, though his comments on the moral and political issues show the clear thinking and fine writing that we expect from him.
P. B. MooN 